\title{
The Relativistic Beaming Model and Superluminal Motions ${ }^{1}$
}

\author{
J. H. Fan
}

Center for Astrophysics, Guangzhou Normal University, Guangzhou 510400, China

G. Z. Xie, Y.H. Zhang, and Y.P. Qin

Yunnan Observatory, The Chinese Academy of Science, Kunming, China

\section{Introduction}

In this paper, we consider a compilation of 55 objects with known superluminal motions (SM), and whose flux density (X-ray, optical, radio), core dominance parameter $(R)$, superluminal velocity, and radio Doppler factor $\left(\delta_{R}\right)$ are known. Our study shows that SM is consistent with the beaming model, and the relation

$$
\delta_{\gamma}=\delta_{\mathrm{o}}^{1+\frac{1}{8} \log \left(\nu_{o} / \nu_{\gamma}\right)}
$$

is reasonable. The statistical correlation between superluminal velocity and redshift is a result of selection and the statistical correlation between $R$ and brightness temperature $\left(T_{o b}\right)$ is actually a reflection of the correlations between $\delta, R$, and $T_{o b}$ for objects with SM. Up to now, 59 objects have been reported to have SM, but for reasons discussed elsewhere (Vermeulen \& Cohen 1994), only 55 are considered here.

\section{Acceleration Model}

We proposed a formula between Doppler factor and frequency (the 'acceleration model', as we call it),

$$
\delta_{\gamma}=\delta_{\mathrm{o}}^{1+\frac{1}{8} \log \left(\nu_{\rho} / \nu_{\gamma}\right)}
$$

which is true for BL Lac objects (Fan et al. 1993, 1994) and Seyfert galaxies (Xie et al. 1995), and has been confirmed (Fan \& Xie 1996). Here, we give another confirmation of SM.

From our previous paper (Fan et al. 1996), we have

$$
\delta_{R}=\delta_{\mathrm{o}}^{1.93 \pm 0.22},
$$

which is consistent with the theoretical result $\delta_{R}=\delta_{0}^{1.67}$ obtained from eq. (1) with $\nu_{R}=5 \mathrm{GHz}$, and $\nu_{\mathrm{o}}=10^{14.74} \mathrm{~Hz}$. So, the acceleration model is reasonable.

\footnotetext{
${ }^{1}$ This work is supported by the National Natural Science Foundation of China and the Natural Science Foundation of Guangdong Province.
} 


\section{Statistical Results}

When linear regression analysis is applied to the data, following statistical results are obtained at a confidence level of greater than $99 \%: \log R-\log \delta, \log R-$ $\log T_{o b}, \log S_{r}-\log \delta$, and $\log \beta-\log z$. However, when the method of Padovani (1992) is used, we find that there are no longer correlations for $\log R-\log T_{o b}$ and $\log \beta-\log z$; these statistical correlations are actually a reflection of the correlations between $\delta, R$ and $T_{o b}$, and a result of selection, respectively.

\section{References}

Fan, J.H., et al. 1993, ApJ, 415, 113.

Fan, J.H., et al. 1994, ApSS, 213, 305.

Fan, J.H., et al. 1996, A\&AS, 116, in press.

Fan, J.H., \& Xie, G. Z. 1996, A\&A, 306, 55.

Padovani, P. 1992, A\&A, 256, 399.

Vermeulen, R. C., \& Cohen, M. H. 1994, ApJ, 430, 467.

Xie, G.Z., et al. 1995, ApJ, 454, 50. 